\title{
Clinicians' attitudes towards clinical trials of cancer therapy
}

\author{
E Ford $^{*, 1}$, V Jenkins', L Fallowfield', N Stuart ${ }^{2}$, D Farewell ${ }^{3}$ and V Farewell ${ }^{4}$ \\ 'Cancer Research UK Psychosocial Oncology Group, Brighton and Sussex Medical School, University of Sussex, Falmer, Brighton BNI 9QG, UK; ${ }^{2}$ School of \\ Medical Sciences, Brigantia Building, University of Bangor, Penrallt Road, Bangor, Wales LL57 2AS, UK; ${ }^{3}$ Department of Primary Care and Public Health, \\ Cardiff University School of Medicine Neuadd Meirionnydd, Heath Park, Cardiff CFI 4 4YS, UK; ${ }^{4}$ MRC Biostatistics Unit, Institute of Public Health, \\ University Fonvie Site, Robinson Way, Cambridge CB2 OSR, UK
}

\begin{abstract}
BACKGROUND: Patient accrual into cancer clinical trials remains at low levels. This survey elicited attitudes and practices of cancer clinicians towards clinical trials.

METHOD: The 43-item Clinicians Attitudes to Clinical Trials Questionnaire was completed by participants in an intervention study aimed at improving multi-disciplinary involvement in randomised trials. Responses from 13 items were summed to form a researchorientation score.

RESULTS: Eighty-seven clinicians (78\%) returned questionnaires. Physicians, more often than surgeons, chose to prioritise prolonging a patient's life, recruited $\geqslant 50 \%$ of patients into trials and attended more research-focussed conferences. Clinicians at specialist centres were more positive about trials with no-treatment arms than those at district general hospitals, more likely to believe clinician, rather than patient reluctance to participate was the greater obstacle to trial accrual, and preferred national and international to local recognition. Clinicians belonging to breast and colorectal teams were less disappointed about not enrolling patients in trials and more accepting of no-treatment arm trials. Research orientation was higher in physicians than surgeons and higher in specialist centres than district hospitals.

CONCLUSIONS: This study provides greater understanding of clinicians' attitudes to trials. Results have been used to inform training interventions for clinicians targeting the problem of low and selective accrual.

British Journal of Cancer (201I) 104, 1535- 1543. doi:10.1038/bjc.201 I.II9 www.bjcancer.com

Published online 12 April 2011

(C) 20II Cancer Research UK
\end{abstract}

Keywords: clinical trials; oncologists; attitudes; recruitment; cancer therapy

The randomised controlled trial (RCT) remains the gold-standard by which new cancer treatments are assessed and through which therapeutic progress is made. Despite the need to develop new cancer therapies, accrual of patients into such trials remains low with fewer than $5 \%$ of cancer patients participating (Tejeda et al, 1996; Christian and Trimble, 2003; Murthy et al, 2004; Somkin et al, 2005; Elting et al, 2006). Even in centres running trials suitable for a particular patient group such as women with limited, local breast cancer or other solid tumours, participation of those eligible is only $12-30 \%$ (Elting et al, 2006; Maslin-Prothero, 2006). As well as increasing the time needed to complete studies this low recruitment means that randomised patients may differ significantly from those who do not take part. This risks undermining the study results by making it difficult to draw generally applicable conclusions (Elting et al, 2006). Several US and European organisations acknowledge that slow accrual hampers the timely release of new therapies into the clinic. For example, only about $60 \%$ of US National Cancer Institute (NCI)-sponsored trials are completed and published (Nass et al, 2010). In addition, certain patient populations are under-represented, resulting in the recent development of several national and international programmes to

*Correspondence: Dr E Ford; E-mail: e.ford@sussex.ac.uk Received 31 January 2011; revised II March 201 I; accepted I4 March 20 I I; published online I2 April 20 I I address the problem of slow and selective recruitment (EDICT, 2008; Lally and Crome, 2010; Seifer et al, 2010).

Studies have found that the majority of clinicians favour clinical trials, viewing them as a source of high-quality patient care, and as a benefit to themselves, their institutions, and to society (Weinberg et al, 2004; Somkin et al, 2005). However, there are two main problems needing attention if trial participation is to be increased: first that clinicians do not offer trials to all eligible patients (Kaas et al, 2005), and second that some patients offered seemingly appropriate trials refuse participation (Siminoff et al, 2000). Reducing clinician gate-keeping and broadening the range of patients to whom trials are offered is challenging, but improving clinicians' communication skills when explaining trials has the potential to resolve patients' concerns and increase the likelihood of their participation. A review of NCI-sponsored trials noted the importance of changing physicians' perspectives so that they will be more likely to encourage their patients to participate in clinical trials (Nass et al, 2010). Understanding more about clinicians' attitudes and practices in this area is necessary and timely, if recruitment is to be improved.

Research shows that clinicians often adopt stringent, idiosyncratic criteria when selecting the patients to whom they offer trials, over and above the criteria delineated in trial protocols. In particular, some clinicians only select patients with even better health status and prognosis than demanded by the protocol (Antman et al, 1985; Hjorth et al, 1992; Rahman et al, 1997; Cottin et al, 1999; Siminoff et al, 2000) or choose not to offer trial 
participation on putative compassionate grounds (Fayter et al, 2007). Clinicians may hesitate to inform patients of trials, based on their own attitudes and beliefs about a patient's willingness to participate, ability to understand the trial, or adhere to the protocol (EDICT, 2008). This is despite $83 \%$ of patients being potentially willing to participate if given full trial information (Jenkins et al, 2010). Other clinicians are reluctant to enrol patients in trials if the treatment concerned may result in extra side effects (White et al, 2008). If older and sicker patients are not included in clinical trials then trial participants are likely to have better outcomes than would be seen were the new treatments offered in normal clinical practice.

Perhaps unsurprisingly, assessments of clinicians' attitudes show that many tend to be clinically, rather than research oriented, believing that individual benefit for their patient is more important than improving future therapy (Caldwell et al, 2005). Some indicate that the main reason for including their patients in trials is so that they receive state of the art treatment (Joffe and Weeks, 2002), and trials focussing on quality of life outcomes tend to have better accrual rates (Hjorth et al, 1996).

A lack of confidence in explaining clinical trials to patients and obtaining informed consent is a barrier to some clinicians (Tournoux et al, 2006; Fayter et al, 2007). Clinicians are reticent to enrol patients who may have difficulty understanding what the trial involves (Weinberg et al, 2004). If the trial arms are not equally attractive or there is a no-treatment arm, this too may prove a disincentive (Maslin-Prothero, 2006). Furthermore, if the protocol is overly arduous to implement (e.g., requiring substantial time or resource commitments from the clinician or their organisation) clinicians are unlikely to recruit a large number of patients (Hjorth et al, 1996; Ellis et al, 1999; Grunfeld et al, 2002; Weinberg et al, 2004; Tournoux et al, 2006; Fayter et al, 2007). Some patients also may have a problem with understanding the concept of randomisation (Jenkins et al, 2002) and may struggle to accept clinical equipoise, for example, in the case of a placebo or no-treatment arm trial (Robinson et al, 2005). Furthermore, clinicians may explain these concepts in ways that patients may find hard to understand or even find threatening (Jenkins et al, 2002).

Despite these barriers, the majority of medical and clinical/ radiation oncologists do report enrolling patients into clinical trials (Fallowfield et al, 1997). Interestingly, different specialties within oncology have different accrual rates, although which specialties accrue more is not consistent. In one survey of UK clinicians, medical oncologists generally placed more emphasis on research than did surgical or clinical oncologists, and felt greater pressure to participate in trials (Fallowfield et al, 1997). In Australia, medical and surgical oncologists participated more in clinical trials than radiation oncologists (Ellis et al, 1999). In the United States, medical and paediatric oncologists reported the lowest rates of patient enrolment in trials (Joffe and Weeks, 2002), although a more recent study showed greater recruitment among medical and radiation oncologists than among surgeons (Klabunde et $a l, 2011)$. A further study found no differences in attitudes towards clinical trials between surgeons and physicians, although this was not limited to oncologists (McCulloch et al, 2005). An understanding of how differences between specialists affect trial recruitment may help promote accrual across specialties. Some suggested reasons for differences are type of education, concept of medicine, individual or professional aims, affiliation with academic or research groups, or particular working environment (Castel et al, 2006), while Klabunde et al (2011) suggest that specialty type, involvement in teaching, affiliation with a Community Clinical Oncology Programme or with a National Cancer Institute-designated cancer centre were important.

Greater understanding of clinicians' attitudes to clinical trials is needed to target the problem of low and selective accrual (Castel et al, 2006). The present survey elicited clinician attitudes towards clinical trials in their practice using a modified version of the Physician Orientation Profile (Taylor and Kelner, 1987). The aims were to assess clinician responses for differences by specialty, type of hospital, type of team, and geographic region and to estimate a research-orientation score for each clinician, again looking for differences between groups. The data used for this study are a component of a large Cancer Research UK funded prospective study examining multidisciplinary team members' communication skills and involvement in clinical trials; primarily randomised, controlled, phase 3 trials. The main study examined different aspects of trial recruitment, including involvement of individual team members in clinical trials; assessment of the clarity of health professionals' communication by patients recruited into clinical trials, and attitudes towards RCTs (Figure 1). The attitudes of patients to RCTs (Jenkins et al, 2010) and that of clinicians to RCTs were collected for each multi-disciplinary team (MDT) to provide an evidence-based argument for encouraging clinicians to consider approaching more patients about trials. The clinician data are presented here.

\section{MATERIALS AND METHODS}

\section{Sample}

Recruitment of clinicians (senior doctors) within oncology MDTs (also known as interdisciplinary tumour boards) was a joint effort between the Cancer Research UK Psychosocial Oncology Group and the Wales Cancer Trials Network. Twenty-two MDTs throughout Wales participated in the larger communication study. The study had ethical approval from the South East Wales Local Research Ethics Committee (ref: 07/WSE03/17). Teams were randomised to receive communication training or to go on a waiting list. Consultant (attending) surgeons, oncologists, chest physicians, and haematologists were asked to complete questionnaires examining their attitudes to trials and their involvement in trials before randomisation.

\section{Materials}

The Clinicians' Attitudes to Clinical Trials of Cancer Therapy Questionnaire is a modified version of the 30-item, binary option, Physician's Orientation Profile (Taylor and Kelner, 1987) and has been used before by this research group (Fallowfield et al, 1997). Items on this questionnaire are classified into five subscales assessing various aspects of clinicians' attitudes towards their clinical and scientific work, specifically primary allegiance, professional activities, decision-making under uncertainty, perceived professional rewards, and peer group influence. The questionnaire used in this study had 43 items and is very similar to the 45-item questionnaire used by Fallowfield et al (1997). Professional information (specialty, MDT cancer site, etc.) was also collected for each clinician.

\section{Derivation of research-orientation score}

Two senior researchers (LF and VJ) independently chose items from the questionnaire on the basis of their face validity for the construct 'Research Orientation'. Each researcher independently chose 18 items from the original 43 with $100 \%$ consistency. The items were recoded where necessary to provide binary responses ( 0 out of 1 ) with 1 indicative of a research orientation. The chosen items were subjected to statistical scrutiny to assess their factorial validity and reliability. Classical principal components analysis (PCA) was undertaken based on the binary responses to these 18 questions (D'Agostino Snr, 2005) with the pragmatic aim of identifying items, which were well correlated and could be hypothesised to represent one or more underlying factors or constructs. Following PCA, a scree plot suggested a single 


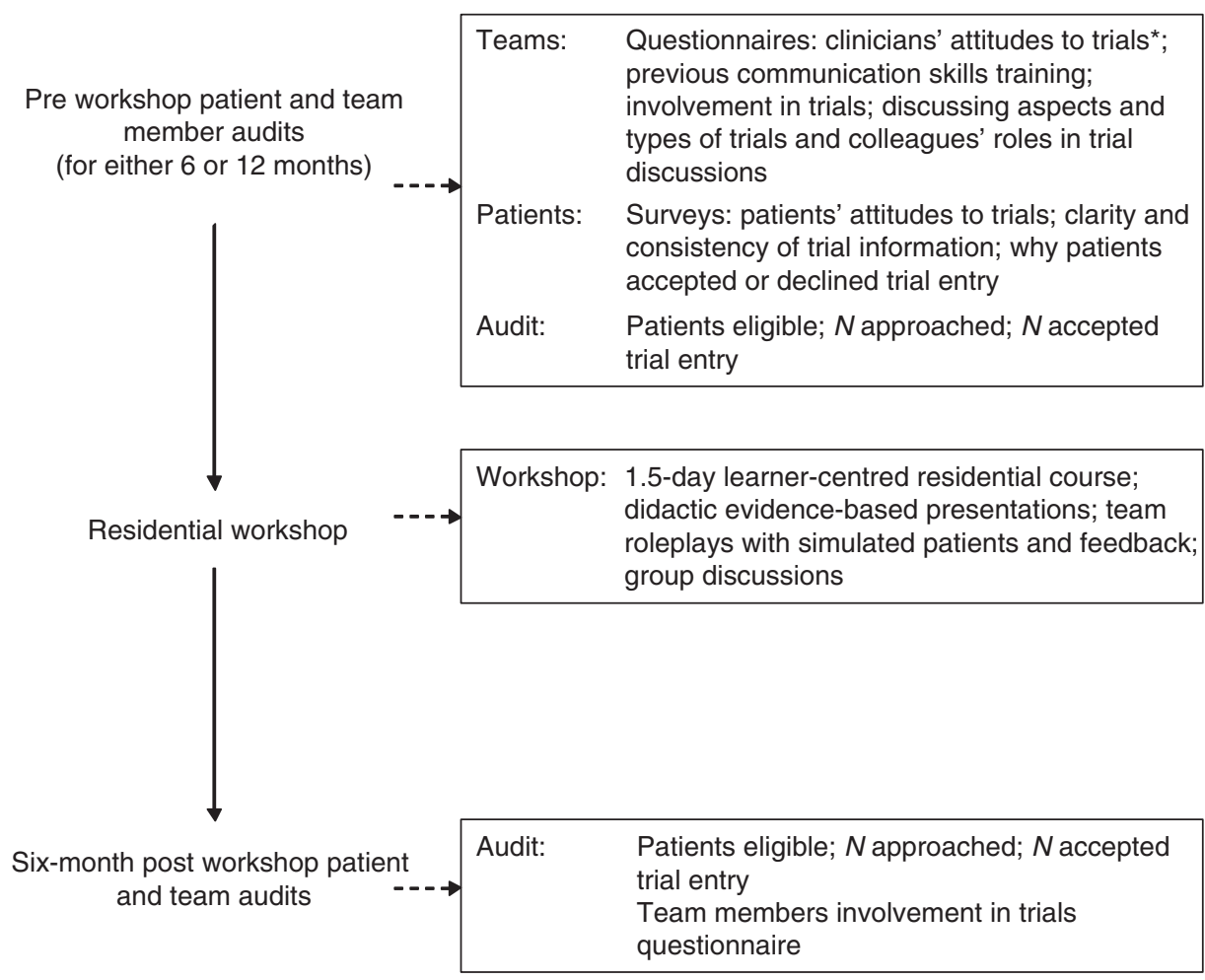

Figure I Overall 'Teams Talking About Trials' project. *Data presented in this paper.

component solution was most suitable for these items (first component eigenvalue $5.79,32.15 \%$ of variance; second component $2.03,11.29 \%$ of variance). Items $6,7,8,12,13,16,17,27,28$, $31,35,36$, and 40 loaded onto the first component with loadings from 0.47 to 0.83 . Item 11 loaded at 0.32 and items $5,22,32,43$ loaded at $<0.30$.

All 18 items were then examined for internal consistency. Cronbach's $\alpha$ was found to be 0.83 , and low item-total correlations were found for items $5(0.02), 11(0.27), 22(0.13), 32(0.13)$, and $43(0.20)$, suggesting that $\alpha$ would increase by removing these items. Items $5,11,22,32$, and 43 were removed and Cronbach's $\alpha$ recalculated for the 13 items $(\alpha=0.89)$. A second PCA on these remaining 13 items showed again that these items loaded onto one component explaining $44.4 \%$ of the variance (loadings $0.49-0.84$ ). The responses from the remaining 13 items were summed to form a research-orientation score.

\section{Analysis}

Question response was linked to possible explanatory variables through logistic regression. Random effects were used to account for within-team correlations, although in general these correlations were low. As the number of clinicians returning questionnaires was relatively small compared with the number of questions, and with conservatism introduced because of within-team correlations, there is little scope for multiplicity adjustments. Thus, significance levels related to individual questions should be treated with caution and seen as primarily providing an ordering of observed discriminatory ability. The analysis of the derived researchorientation score, which represented the sum of 13 questions, was based on continuation ratio ordinal regression analysis, with use of a complementary log-log link. The more flexible continuation ratio model was preferred to a binomial regression because of the variability between questions in the proportion of 'research oriented' answers.

\section{RESULTS}

Out of the 111 clinicians approached, 87 (78.4\%) returned questionnaires. The sample was formed of 47 surgeons, 28 oncologists, 9 haematologists, and 3 chest physicians. These clinicians belonged to MDTs for the following cancers: breast $(25 ; 31 \%)$, colorectal $(15 ; 17 \%)$, gynaecology $(8 ; 9 \%)$, haematology $(10 ; 11.5 \%)$, lung $(6 ; 7 \%)$, lymphoma $(3 ; 3 \%)$, upper gastrointestinal $(10 ; 11.5 \%)$, and urology $(10 ; 11.5 \%)$. Responses to each item on the questionnaire, and differences in responses by separate groupings (physician vs surgeon; specialist hospital (large teaching or city hospital) $v s$ district general hospital (DGH) (community hospital); breast and colorectal teams $v s$ others) are shown in Table 1.

\section{Differences by specialty}

Table 2 shows the questionnaire items that best distinguished between physicians (oncologists, haematologists, and chest physicians) and surgeons. Physicians more frequently endorsed prolonging a patient's life over improving quality of life. They were also more likely to report recruiting $50 \%$ or more of their patients into clinical trials and attending conferences focussed on research rather than clinical issues. These differences suggest physicians may be more research focussed than their surgical counterparts.

\section{Differences by centre}

Table 3 shows the questionnaire items best differentiating clinicians who worked in a specialist cancer centre from those who worked in a DGH. Those working at a specialist centre were less reluctant to randomise patients into trials with no-treatment arms, more likely to believe that clinician reluctance (as opposed to patient reluctance) to participate was a major obstacle to trial accrual, and were more likely to wish to be well known among 
Table I Differences in response in physicians vs surgeons, specialist hospital vs district general and breast and colorectal teams vs other teams

\begin{tabular}{|c|c|c|c|c|c|c|c|}
\hline & $\begin{array}{c}\text { Overall } \\
\text { Sample (\%) }\end{array}$ & $\begin{array}{l}\text { Physician (\%) } \\
\quad(n=40)\end{array}$ & $\begin{array}{l}\text { Surgeon (\%) } \\
\quad(n=47)\end{array}$ & $\begin{array}{c}\text { DGH (\%) } \\
(n=33)\end{array}$ & $\begin{array}{l}\text { Specialist (\%) } \\
\quad(n=54)\end{array}$ & $\begin{array}{l}\text { Breast/colorectal } \\
\quad(\%)(n=40)\end{array}$ & $\begin{array}{l}\text { Other cancer typ } \\
\text { (\%) }(n=47)\end{array}$ \\
\hline \multicolumn{8}{|c|}{ Q1. Ideally, clinicians are able to increase survival and improve the quality of patients' lives. In cases where only one can be achieved at the cost of the other, I feel more satisfied when I can: } \\
\hline (a) improve patients' quality of life & $7 /(83.5 \%)$ & $29(74.3)$ & $42(91.3)$ & $27(84.4)$ & $44(83.0)$ & $32(82.1)$ & $39(84.8)$ \\
\hline (b) prolong patients' lives & $14(16.5 \%)$ & $10(25.6)$ & $4(8.7)$ & $5(15.6)$ & $9(17.0)$ & $7(17.9)$ & $7(15.2)$ \\
\hline \multicolumn{8}{|c|}{ Q2. If a patient refuses to participate in a randomised clinical trial that I suggest, I would: } \\
\hline (a) treat the patient off the study & $87(100 \%)$ & - & - & - & - & - & - \\
\hline (b) refer the patient to another clinician & $0(0 \%)$ & - & - & - & - & - & - \\
\hline \multicolumn{8}{|l|}{ Q3. In general, when I initiate a treatment for cancer, I am: } \\
\hline (a) optimistic that the treatment will work & $80(93.0 \%)$ & $38(97.4)$ & $42(89.4)$ & $31(93.9)$ & $49(92.5)$ & $37(92.5)$ & $43(93.4)$ \\
\hline (b) pessimistic that the treatment will work & $6(7.0 \%)$ & I (2.6) & $5(10.6)$ & $2(6.1)$ & $4(7.5)$ & $3(7.5)$ & $3(6.5)$ \\
\hline \multicolumn{8}{|c|}{ Q4. In my hospital the pressure to participate in a randomised clinical trial is relatively: } \\
\hline (a) low & $58(68.2 \%)$ & $2 \mathrm{I}(53.8)$ & $37(80.4)$ & $26(78.8)$ & $32(61.5)$ & $29(74.4)$ & $29(63.0)$ \\
\hline (b) high & $27(31.8 \%)$ & $18(46.2)$ & $9(19.6)$ & $7(21.2)$ & $20(38.5)$ & $10(25.6)$ & $17(37.0)$ \\
\hline \multicolumn{8}{|c|}{ Q5. I enter the following amount of my potentially eligible patients into randomised clinical trials: } \\
\hline (a) under $50 \%$ & $66(75.9 \%)$ & $25(62.5)$ & $4 \mid(87.2)$ & $27(81.8)$ & $39(72.2)$ & $31(77.5)$ & $35(74.5)$ \\
\hline (b) $50 \%$ or more & $21(24.1 \%)$ & $15(37.5)$ & $6(12.8)$ & $6(18.2)$ & $15(27.8)$ & $9(22.5)$ & $12(25.5)$ \\
\hline \multicolumn{8}{|l|}{ Q6. My primary commitment is to: } \\
\hline (a) future generations of patients (society) & $9(10.7 \%)$ & $4(10.3)$ & 5 (II.I) & $3(9.1)$ & $6(11.8)$ & 7 (18.4) & $2(4.3)$ \\
\hline (b) present patients (individuals) & $75(89.3 \%)$ & 35 (89.7) & 40 (88.9) & $30(90.9)$ & 45 (88.2) & $31(81.6)$ & $44(95.6)$ \\
\hline \multicolumn{8}{|c|}{ Q7. When faced with a controversial treatment decision, I feel most comfortable when: } \\
\hline (a) I make the decisions & II (1 $2.8 \%)$ & $4(10.3)$ & 7 (14.9) & $4(12.1)$ & 7 (13.2) & $6(15.0)$ & $5(10.9)$ \\
\hline (b) the decisions are made by the trial protocol & $8(9.3 \%)$ & $4(10.3)$ & 4 (8.5) & $2(6.1)$ & $6(11.3)$ & $3(7.5)$ & $5(10.9)$ \\
\hline (c) the decision is made by the Multi-Disciplinary Meeting & $67(77.9 \%)$ & $31(79.5)$ & $36(76.6)$ & $27(81.8)$ & $40(75.5)$ & $31(77.5)$ & $36(78.3)$ \\
\hline \multicolumn{8}{|c|}{ Q8. Currently, I am the principal investigator on one or more research grants: } \\
\hline (a) no & $55(63.2 \%)$ & $22(55.0)$ & $33(70.2)$ & $26(78.8)$ & $29(53.7)$ & $27(67.5)$ & $28(59.6)$ \\
\hline (b) yes & 32 (36.7\%) & $18(45.0)$ & $14(29.8)$ & 7 (21.2) & $25(46.3)$ & $13(32.5)$ & $19(40.4)$ \\
\hline \multicolumn{8}{|l|}{ Q9. In my hospital, doctors are given more reward for. } \\
\hline (a) clinical skills with patients & $63(79.7 \%)$ & $28(75.7)$ & $35(83.3)$ & $29(87.9)$ & $34(73.9)$ & $29(82.9)$ & $34(77.3)$ \\
\hline (b) contributing to scientific knowledge & $16(20.3 \%)$ & $9(24.3)$ & $7(16.7)$ & $4(12.1)$ & $12(26.1)$ & $6(17.1)$ & $10(22.7)$ \\
\hline \multicolumn{8}{|c|}{ Q10. When a patient on a protocol relapses or progresses and the protocol dictates additional treatment that the patient does not want, I: } \\
\hline (a) encourage the patient to stay on the trial & $28(33.7 \%)$ & II (28.2) & $17(38.6)$ & II (33.3) & $17(34.0)$ & $13(35.1)$ & $15(32.6)$ \\
\hline (b) remove the patient from the trial & $55(66.3 \%)$ & $28(71.8)$ & $27(61.4)$ & $22(66.7)$ & $33(66.0)$ & $24(64.9)$ & $31(67.4)$ \\
\hline \multicolumn{8}{|l|}{ Q11. In general, patients are referred to me because of my: } \\
\hline (a) research activities & $3(3.5 \%)$ & $2(5.1)$ & | (2.1) & I (3.0) & $2(3.8)$ & $2(5.0)$ & I (2.2) \\
\hline (b) clinical reputation & $83(96.5 \%)$ & $37(94.9)$ & $46(97.9)$ & $32(97.0)$ & $51(96.2)$ & $38(95.0)$ & $45(97.8)$ \\
\hline \multicolumn{8}{|c|}{ QI2. I participate more actively in professional organisations that are based on: } \\
\hline (a) my clinical speciality & 74 (87.1\%) & $29(76.3)$ & 45 (95.7) & $32(97.0)$ & $42(80.8)$ & 35 (89.7) & $39(84.8)$ \\
\hline (b) my research activities & II (12.9\%) & 9 (23.7) & $2(4.3)$ & 1 (3.0) & $10(19.2)$ & $4(10.3)$ & 7 (15.2) \\
\hline \multicolumn{8}{|c|}{ Q13. The time I devote to publications, lectures and research commitments, compared with clinical work, is relatively: } \\
\hline (a) low & $74(86.0 \%)$ & 31 (79.5) & $43(91.5)$ & $32(97.0)$ & $42(79.2)$ & $34(85.0)$ & $40(87.0)$ \\
\hline (b) high & $12(14.0 \%)$ & $8(20.5)$ & $4(8.5)$ & I (3.0) & II (20.8) & $6(15.0)$ & $6(13.0)$ \\
\hline Q14. The need for detailed monitoring of individual clinicians' activities deter & $m$ participating in & randomised clinical & al trials: & & & & \\
\hline (a) no & $72(82.8 \%)$ & $37(92.5)$ & $35(74.5)$ & $24(72.7)$ & $48(88.9)$ & $32(80.0)$ & $40(85.1)$ \\
\hline (b) yes & $15(17.2 \%)$ & $3(7.5)$ & $12(25.5)$ & $9(27.3)$ & $6(11.1)$ & $8(20.0)$ & $7(14.9)$ \\
\hline Q15. When a potentially eligible patient chooses not to enrol on a trial that & suggested, l: & & & & & & \\
\hline (a) often feel disappointed & $26(30.2 \%)$ & II (27.5) & $15(32.6)$ & $10(30.3)$ & $16(30.2)$ & $6(15.4)$ & $20(42.6)$ \\
\hline (b) seldom feel disappointed & $60(69.8 \%)$ & $29(72.5)$ & $31(67.4)$ & $23(69.7)$ & $37(69.8)$ & $33(84.6)$ & $27(57.4)$ \\
\hline Q16. I devote $a$ lot of time to educating other clinicians about rc & sed clinical tria & & & & & & \\
\hline (a) no & $60(69.0 \%)$ & $21(52.5)$ & $39(83.0)$ & $27(81.8)$ & $33(61.1)$ & $27(67.5)$ & $33(70.2)$ \\
\hline (b) yes & $27(31.0 \%)$ & $19(47.5)$ & $8(17.0)$ & $6(18.2)$ & $21(38.9)$ & $13(32.5)$ & $14(29.8)$ \\
\hline QI7. Frequent publications are important to my career advancer & & & & & & & \\
\hline (a) agree & $45(52.3 \%)$ & $21(52.5)$ & $24(52.2)$ & $14(42.4)$ & $31(58.5)$ & $22(56.4)$ & $23(48.9)$ \\
\hline (b) disagree & $41(47.7 \%)$ & 19 (47.5) & $22(47.8)$ & $19(57.6)$ & $22(41.5)$ & $17(43.6)$ & $24(51.1)$ \\
\hline Q 18. When a protocol includes a treatment that is more aggressive than I $\mathrm{v}$ & ually give to similc & r non-trial patien & & & & & \\
\hline (a) I am often reluctant to participate & $27(31.4 \%)$ & $14(35.0)$ & $13(28.3)$ & $8(24.2)$ & $19(35.8)$ & | | (28.2) & $16(34.0)$ \\
\hline (b) it makes no difference & $59(68.6 \%)$ & $26(65.0)$ & $33(71.7)$ & $25(75.8)$ & $34(64.2)$ & $28(71.8)$ & $31(66.0)$ \\
\hline Q19. I am reluctant to participate in a trial that may randomise the patient & treatment' group & & & & & & \\
\hline (a) agree & $18(20.7 \%)$ & $5(12.5)$ & $13(27.7)$ & | | (33.3) & $7(13.0)$ & $14(35.0)$ & $4(8.5)$ \\
\hline (b) disagree & $69(79.3 \%)$ & $35(87.5)$ & $34(72.3)$ & $22(66.7)$ & $47(87.0)$ & $26(65.0)$ & $43(91.5)$ \\
\hline Q20. After being randomised, if a patient refuses the treatment to which he & has been assigned & & & & & & \\
\hline (a) I accept the patient's decision & $71(81.6 \%)$ & $28(70.0)$ & $43(9 \mid .5)$ & $28(84.8)$ & $43(79.6)$ & $35(87.5)$ & $36(76.6)$ \\
\hline (b) I make every effort to keep the patient on the trial & $16(18.4 \%)$ & $12(30.0)$ & $4(8.5)$ & $5(15.1)$ & II (20.4) & $5(12.5)$ & II (23.4) \\
\hline Q21. Overall I feel the quality of patient care: & & & & & & & \\
\hline (a) increases when patient is in a clinical trial & $83(98.8 \%)$ & $40(100)$ & $43(97.7)$ & $30(96.8)$ & $53(100)$ & $38(97.4)$ & $45(100)$ \\
\hline (b) decreases when patient is in a clinical trial & I (1.2\%) & $0(0)$ & I (2.3) & । (3.2) & $0(0)$ & I (2.6) & $0(0)$ \\
\hline
\end{tabular}


Table I (Continued)

\begin{tabular}{|c|c|c|c|c|c|c|c|}
\hline & $\begin{array}{c}\text { Overall } \\
\text { Sample (\%) }\end{array}$ & $\begin{array}{l}\text { Physician (\%) } \\
\quad(n=40)\end{array}$ & $\begin{array}{l}\text { Surgeon (\%) } \\
\quad(n=47)\end{array}$ & $\begin{array}{l}\text { DGH (\%) } \\
(n=33)\end{array}$ & $\begin{array}{c}\text { Specialist (\%) } \\
(n=54)\end{array}$ & $\begin{array}{l}\text { Breast/colorectal } \\
(\%)(n=40)\end{array}$ & $\begin{array}{l}\text { Other cancer type } \\
(\%)(n=47)\end{array}$ \\
\hline \multicolumn{8}{|c|}{ Q22. When published data and my clinical experience conflict, I am more likely to rely on: } \\
\hline (a) my clinical experience & $49(59.0 \%)$ & $20(52.6)$ & $29(64.4)$ & $22(71.0)$ & $27(5 \mid .9)$ & $24(6 \mid .5)$ & $25(56.8)$ \\
\hline (b) published data & $34(41.0 \%)$ & $18(47.4)$ & $16(35.6)$ & $9(29.0)$ & $25(48.1)$ & $15(38.5)$ & $19(43.2)$ \\
\hline \multicolumn{8}{|l|}{ Q23. The more frequent obstacle to the successful completion of a clinical trial is: } \\
\hline (a) clinicians' reluctance to participate & $57(67.1 \%)$ & $27(67.5)$ & 30 (66.7) & $17(53.1)$ & $40(75.4)$ & $26(68.4)$ & $31(66.0)$ \\
\hline (b) patients' reluctance to participate & $28(32.9 \%)$ & $13(32.5)$ & $15(33.3)$ & $15(46.9)$ & $13(24.5)$ & $12(31.6)$ & $16(34.0)$ \\
\hline \multicolumn{8}{|c|}{ Q24. If written informed consent were not required, I would approach more patients to enter clinical trials: } \\
\hline (a) true & $16(18.4 \%)$ & $5(12.5)$ & II (23.4) & $5(\mid 5.1)$ & || (20.4) & $10(25.0)$ & $6(12.8)$ \\
\hline (b) false & $71(81.6 \%)$ & $35(87.5)$ & $36(76.6)$ & $28(84.8)$ & $43(79.6)$ & $30(75.0)$ & $41(87.2)$ \\
\hline \multicolumn{8}{|c|}{ Q25. The opinions of the referring clinician regarding randomised clinical trials affects my decision to approach an eligible patient: } \\
\hline (a) true & $14(16.3 \%)$ & $5(12.5)$ & $9(19.6)$ & $5(15.1)$ & $9(17.0)$ & $8(20.5)$ & $6(12.8)$ \\
\hline (b) false & $72(83.7 \%)$ & $35(87.5)$ & $37(80.4)$ & $28(84.8)$ & $44(83.0)$ & $31(79.5)$ & $41(87.2)$ \\
\hline \multicolumn{8}{|c|}{ Q26. The thought of having to spell out all the details of a trial to eligible patients discourages me from approaching them to participate: } \\
\hline (a) true & $20(23.0 \%)$ & $7(17.5)^{\circ}$ & $13(27.7)$ & $9(27.3)$ & || (20.4) & $13(32.5)$ & $7(14.9)$ \\
\hline (b) false & $67(77.0 \%)$ & $33(82.5)$ & $34(72.3)$ & $24(72.7)$ & $43(79.6)$ & $27(67.5)$ & $40(85.1)$ \\
\hline \multicolumn{8}{|l|}{ Q27. It is more important for me to be well-known among: } \\
\hline (a) local colleagues & $56(70.0 \%)$ & $24(63.2)$ & $32(76.2)$ & $28(90.3)$ & $28(57.1)$ & $28(82.4)$ & $28(60.9)$ \\
\hline (b) national/international colleagues & $24(30.0 \%)$ & $14(36.8)$ & $10(23.8)$ & $3(9.7)$ & $21(42.9)$ & $6(17.6)$ & $18(39.1)$ \\
\hline \multicolumn{8}{|c|}{ Q28. I spend the following amount of my time in research-related activities: } \\
\hline (a) less than one-third & 75 (86.2\%) & $31(77.5)$ & $44(93.6)$ & $33(100)$ & $42(77.8)$ & $35(87.5)$ & $40(85.1)$ \\
\hline (b) one-third or more & $12(14.2 \%)$ & $9(22.5)$ & $3(6.4)$ & $0(0)$ & $12(22.2)$ & 5 (12.5) & 7 (14.9) \\
\hline \multicolumn{8}{|c|}{ Q29. A major reason for my participation in randomised clinical trials is that it benefits my institution: } \\
\hline (a) agree & $43(49.4 \%)$ & $20(50)$ & $23(48.9)$ & $14(42.4)$ & $29(53.7)$ & $21(52.5)$ & $22(46.8)$ \\
\hline (b) disagree & $44(50.6 \%)$ & $20(50)$ & $24(51.1)$ & $19(57.6)$ & $25(46.3)$ & $19(47.5)$ & $25(53.2)$ \\
\hline \multirow{2}{*}{\multicolumn{8}{|c|}{$\begin{array}{l}\text { Q30. Overall, participation in a randomised clinical trial is: } \\
\text { (a) an asset to my reputation }\end{array}$}} \\
\hline & $84(100 \%)$ & - & - & - & - & - & - \\
\hline (b) a liability to my reputation & $0(0 \%)$ & - & - & - & - & - & - \\
\hline \multicolumn{8}{|c|}{ Q3I. If I could have only one measure, I would assess how successful I was as a physician by: } \\
\hline (a) my research contributions & 12 (14.0\%) & $8(20.5)$ & 4 (8.5) & I (3.0) & II (20.8) & $5(12.5)$ & 7 (15.2) \\
\hline (b) how I helped individual patients & 74 (86.0\%) & $31(79.5)$ & $43(91.5)$ & $32(97.0)$ & $42(79.2)$ & $35(87.5)$ & $39(84.8)$ \\
\hline \multicolumn{8}{|l|}{ Q32. When I am personally uncertain as to which treatment is best, I am likely to: } \\
\hline (a) enter the patient in a randomised clinical trial if one exists & $74(86.0 \%)$ & $38(95.0)$ & $36(78.3)$ & $27(84.4)$ & $47(87.0)$ & $33(82.5)$ & $41(89.1)$ \\
\hline (b) personally select a treatment & $12(14.0 \%)$ & $2(5.0)$ & $10(21.7)$ & $5(15.6)$ & $7(13.0)$ & $7(17.5)$ & $5(10.9)$ \\
\hline \multicolumn{8}{|l|}{ Q33. When I obtain informed consent: } \\
\hline (a) I allow patient reaction to influence the content of the information given & $50(57.5 \%)$ & $22(55.0)$ & $28(59.6)$ & $22(66.7)$ & $28(5 \mid .9)$ & $25(62.5)$ & $25(53.2)$ \\
\hline (b) I do not vary the content of the information given & $37(42.5 \%)$ & $18(45.0)$ & $19(40.4)$ & II (33.3) & $26(48.1)$ & $15(37.5)$ & $22(46.8)$ \\
\hline Q34. If research activities were to enhance my income, I would enter more patients & in randomised cli & ical trials: & & & & & \\
\hline (a) agree & $28(32.5 \%)$ & $12(30.0)$ & $16(34.8)$ & $12(36.4)$ & $16(30.2)$ & $14(35.9)$ & $14(29.8)$ \\
\hline (b) disagree & $58(67.5 \%)$ & $28(70.0)$ & $30(65.2)$ & $21(63.6)$ & $37(69.8)$ & $25(64.1)$ & $33(70.2)$ \\
\hline Q35. I am more likely to attend a conference that focuses on: & & & & & & & \\
\hline (a) clinical issues & $61(76.3 \%)$ & $23(62.2)$ & $38(88.4)$ & $28(90.3)$ & $33(67.3)$ & $27(77.1)$ & $34(75.6)$ \\
\hline (b) research issues & 19 (23.7\%) & $14(37.8)$ & 5 (11.6) & $3(9.7)$ & $16(32.6)$ & $8(22.9)$ & II (24.4) \\
\hline Q36. In the past 3 years, I have authored/co-authored: & & & & & & & \\
\hline (a) 0 publications & $12(13.7 \%)$ & $9(22.5)$ & $3(6.4)$ & 8 (24.2) & $4(7.4)$ & $7(17.5)$ & $5(10.6)$ \\
\hline (b) I -5 publications & 51 (58.6\%) & $19(47.5)$ & $32(68.1)$ & $21(63.6)$ & $30(55.6)$ & $27(67.5)$ & $24(51.1)$ \\
\hline (c) $6-9$ publications & 14 (16.1\%) & 7 (17.5) & 7 (14.9) & $2(6.1)$ & $12(22.2)$ & $4(10.0)$ & $10(21.3)$ \\
\hline (d) 10 or more publications & $10(11.4 \%)$ & $5(12.5)$ & 5 (10.6) & $2(6.1)$ & 8 (14.8) & $2(5.0)$ & $8(17.0)$ \\
\hline Q37. When informing patients about their prognosis, I find statistics: & & & & & & & \\
\hline (a) helpful & $78(90.7 \%)$ & $36(92.3)$ & $42(89.4)$ & $28(87.5)$ & $50(92.6)$ & $36(90.0)$ & $42(9 \mid .3)$ \\
\hline (b) not helpful & $8(9.3 \%)$ & $3(7.7)$ & $5(10.6)$ & $4(12.5)$ & $4(7.4)$ & $4(10.0)$ & $4(8.7)$ \\
\hline Q38. When making critical and controversial decisions I usually: & & & & & & & \\
\hline (a) seek major input from my patients & $83(96.5 \%)$ & $39(97.5)$ & $44(95.7)$ & $33(100)$ & $50(94.3)$ & $37(94.9)$ & $46(97.9)$ \\
\hline (b) do not seek major input from my patients & $3(3.5 \%)$ & I (2.5) & $2(4.3)$ & $0(0)$ & $3(5.7)$ & $2(5.1)$ & I (2.1) \\
\hline Q39. I think the patient's right to select treatment options is always more important & than the advanc & ment of scientific & nowledge: & & & & \\
\hline (a) true & $73(83.9 \%)$ & $32(80.0)$ & $4 \mid(87.2)$ & $25(75.8)$ & $48(88.9)$ & $33(82.5)$ & $40(85.1)$ \\
\hline (b) false & $14(16.1 \%)$ & $8(20.0)$ & $6(12.8)$ & $8(24.2)$ & $6(11.1)$ & $7(17.5)$ & $7(14.9)$ \\
\hline Q40. If I had to choose, I would say my primary task is: & & & & & & & \\
\hline (a) caring for individual patients & $82(95.3 \%)$ & $36(92.3)$ & $46(97.9)$ & $33(100)$ & $49(92.5)$ & $38(95.0)$ & $44(95.7)$ \\
\hline (b) contributing to scientific knowledge & $4(4.6 \%)$ & $3(7.7)$ & $1(2.1)$ & $0(0)$ & $4(7.5)$ & $2(5.0)$ & $2(4.3)$ \\
\hline Q41. I would rather be somewhat: & & & & & & & \\
\hline (a) too involved with my patients & $75(87.2 \%)$ & $37(92.5)$ & $38(82.6)$ & $26(81.25)$ & $49(90.7)$ & $34(87.2)$ & $4 \mid(87.2)$ \\
\hline (b) too detached from my patients & $11(12.8 \%)$ & $3(7.5)$ & $8(17.4)$ & $6(18.75)$ & $5(9.3)$ & $5(12.8)$ & $6(12.8)$ \\
\hline Q42. When I participate in a randomised clinical trial, it is more likely that I: & & & & & & & \\
\hline (a) increase my patient population & $72(91.1 \%)$ & $36(94.7)$ & $36(87.8)$ & $26(86.7)$ & $46(93.9)$ & $32(91.4)$ & $40(90.9)$ \\
\hline (b) lose patients I might otherwise keep & $7(8.9 \%)$ & $2(5.3)$ & $5(12.2)$ & $4(13.3)$ & $3(6.1)$ & $3(8.6)$ & $4(9.1)$ \\
\hline
\end{tabular}


Table I (Continued)

\begin{tabular}{|c|c|c|c|c|c|c|c|}
\hline & $\begin{array}{c}\text { Overall } \\
\text { Sample (\%) }\end{array}$ & $\begin{array}{l}\text { Physician (\%) } \\
\quad(n=40)\end{array}$ & $\begin{array}{l}\text { Surgeon (\%) } \\
\quad(n=47)\end{array}$ & $\begin{array}{l}\text { DGH (\%) } \\
(n=33)\end{array}$ & $\begin{array}{c}\text { Specialist (\%) } \\
(n=54)\end{array}$ & $\begin{array}{c}\text { Breast/colorectal } \\
(\%)(n=40)\end{array}$ & $\begin{array}{l}\text { Other cancer type } \\
\text { (\%) }(n=47)\end{array}$ \\
\hline \multicolumn{8}{|c|}{ Q43. When there is controversy in the literature as to which treatment is best: } \\
\hline (a) I enter the patient in a clinical trial if one exists & $79(90.8 \%)$ & $38(95.0)$ & $41(87.2)$ & $28(84.8)$ & $51(94.4)$ & $34(85.0)$ & $45(95.7)$ \\
\hline (b) I personally select a treatment for the patient & $8(9.2 \%)$ & $2(5.0)$ & $6(12.8)$ & $5(15.2)$ & $3(5.6)$ & $6(15.0)$ & $2(4.3)$ \\
\hline
\end{tabular}

Abbreviation: $\mathrm{DGH}=$ district general hospital. Items highlighted in bold are used in the research-orientation score.

Table 2 Items distinguishing between physicians and surgeons

\begin{tabular}{lcc}
\hline Questionnaire Item & Physicians & Surgeons \\
\hline Q I. Ideally, clinicians are able to increase survival and improve the quality of patients' lives. In cases where only one can be achieved at the cost of the other, I feel more satisfied when \\
I can: & $29(74 \%)$ & $42(91 \%)$ \\
(a) improve patients' quality of life & $10(26 \%)$ & $4(9 \%)$ \\
(b) prolong patients' lives & & \\
Q5. I enter the following amount of my potentially eligible patients into randomised clinical trials: & $25(62.5 \%)$ & $41(87 \%)$ \\
(a) under 50\% & $15(37.5 \%)$ & $6(13 \%)$ \\
(b) $50 \%$ or more & & $3.29(0.04)$ \\
Q35. I am likely to attend a conference that focuses on: & $23(62 \%)$ & $5(88 \%)$ \\
(a) clinical issues & $14(38 \%)$ & $1.44(0.02)$ \\
(b) research issues &
\end{tabular}

Table 3 Items distinguishing between clinicians in specialist centres and those in district general hospitals

\begin{tabular}{|c|c|c|c|}
\hline Questionnaire Item & DGH & Specialist centre & Beta (sig.) \\
\hline $\begin{array}{l}\text { Q23. The more frequent obstacle to the su } \\
\text { (a) clinicians' reluctance to participate } \\
\text { (b) patients' reluctance to participate }\end{array}$ & $\begin{array}{l}\text { nical trial is: } \\
17(53 \%) \\
15(47 \%)\end{array}$ & $\begin{array}{l}40(76 \%) \\
13(24 \%)\end{array}$ & $-1.00(0.04)$ \\
\hline
\end{tabular}

Abbreviation: DGH = district general hospital.

national or international, rather than local, colleagues. These differences suggest that those at specialist centres may be more research oriented than those at DGHs.

\section{Differences by geographical region}

There were some regional differences in answers to questionnaire items, but after exploration of confounders, it was found that the effect of region (SE $v s \mathrm{~N}$ and SW) disappeared when specialist centre was added to the model.

\section{Differences by type of MDT}

Multi-disciplinary teams were grouped into breast and colorectal teams $v s$ the other teams (haematology, gynaecology, lung, lymphoma, upper gastro-intestinal, and urology). This grouping was chosen because breast and colorectal cancer are areas where there are typically many clinical trials and where recruitment has been common (UK Clinical Research Network, 2010). We hypothesised that MDTs treating such patients may be more favourable to clinical research. Table 4 shows the questionnaire items which best differentiate clinicians by their MDT specialty. These results suggest that breast and colorectal teams may be less disappointed about not enrolling patients in a trial and more accepting of trials with no-treatment arms. Interestingly, clinicians belonging to other types of teams reported stronger publications records and were more desirous of international rather than local recognition.

\section{Research orientation}

Binary responses to questions $6,7,8,12,13,16,17,27,28,31,35$, 36 , and 40 (highlighted in bold in Table 1) were summed to form the research-orientation scale. Full data were available from 74 participants and scores on the scale ranged from 0 to 12 . The numbers of observations with these 13 values were $18,23,7,8,4,4$, $1,1,1,2,3,1$, and 1 , respectively, showing a skew towards the nonresearch orientation end of the scale. Ordinal regression analysis demonstrated that the probability of a high research orientation was greater in physicians vs surgeons $(P<0.001)$ and in respondents from a specialist centre $(P<0.001)$ but little demonstrable effect was associated with MDT type (breast/colorectal vs rest: $P=0.06$ ). Both the physician/surgeon and specialist/DGH centre classifications retained their significance in a multivariate analysis but MDT type demonstrated no relationship with research orientation (Table 5). 
Table 4 Items distinguishing between MDTs

\begin{tabular}{lcc}
\hline Questionnaire item & Breast and colorectal & Others \\
\hline Q15. When a potentially eligible patient chooses not to enrol on a trial that I have suggested I: & $6(15 \%)$ & Beta (sig.) \\
(a) often feel disappointed & $33(85 \%)$ & $20(43 \%)$ \\
(b) seldom feel disappointed & $27(57 \%)$ \\
Q 19. I am reluctant to participate in a trial that may randomise a patient to a 'no treatment' group. & \\
(a) agree & $14(35 \%)$ & $4(09 \%)$ \\
(b) disagree & $26(65 \%)$ & $43(91 \%)$ \\
Q36. In the past 3 years, I have authored/co-authored: & & \\
(a) 0 & $7(17 \%)$ & $5(11 \%)$ \\
(b) I-5 & $27(68 \%)$ & $24(51 \%)$ \\
(c) 6-9 & $4(10 \%)$ & $10(21 \%)$ \\
(d) I0+ & $2(5 \%)$ & $8(17 \%)$ \\
Q27. It is more important for me to be well known among: & & $-1.76(0.005)$ \\
(a) local colleagues & $28(82 \%)$ & $28(61 \%)$ \\
(b) national/international colleagues & $6(18 \%)$ & $18(39 \%)$ \\
\hline
\end{tabular}

Abbreviation: MDT = multi-disciplinary team

Table 5 Multivariate ordinal regression analysis of clinical/researchorientation scale

\begin{tabular}{lccc}
\hline Variable & Coefficient & $\begin{array}{c}\text { Standard } \\
\text { error }\end{array}$ & $\begin{array}{c}\text { Significance } \\
\text { level }\end{array}$ \\
\hline Physician/surgeon & 0.69 & 0.11 & $<0.001$ \\
Specialist/DGH & 0.95 & 0.13 & $<0.001$ \\
Breast/colorectal vs other & 0.03 & 0.11 & 0.78 \\
\hline
\end{tabular}

Abbreviation: $\mathrm{DGH}=$ district general hospital.

\section{DISCUSSION}

Recruitment of patients into clinical trials is essential if progress is to be made in improving cancer care. Despite this, recruitment is often poor with studies slow to complete and with only a small proportion of cancer patients being enrolled in studies. The process of recruitment involves discussion between the patient and their doctor but is preceded by identification of suitable patients fulfilling eligibility criteria and then the doctor's decision to offer the patient an appropriate trial. The attitude of doctors is therefore a critical part of the enrolment process.

This study evaluated doctors' attitudes to clinical research and to clinical trial recruitment. Using the Clinicians' Attitudes to Clinical Trials of Cancer Therapy Questionnaire the attitudes of 87 clinicians with a range of specialties, tumour site interests, geographical location, and affiliations were assessed. The questionnaire also allowed clinicians to be allocated a research orientation score.

The results indicate that research orientation was greater in physicians than surgeons, with physicians more likely to enter patients into trials and more likely to attend research-focussed conferences. The lesser research orientation found in surgeons may reflect the paucity of surgical trials available and the difficulties encountered recruiting patients into a surgical trial. One study of US breast cancer surgeons showed that $26 \%$ did not discuss trials with any breast cancer patients, and a further $39 \%$ discussed trials with fewer than $10 \%$. Surgeons identified inadequate infrastructure and lack of time as significant deterrents in trial participation (Schroen and Brenin, 2008). Klabunde et al (2011) also show significantly lower clinical trial recruitment among surgeons compared with oncologists although surgical oncologists were more likely to participate in clinical trials than general surgeons. Furthermore, surgeons showed the same pattern as physicians with increased trial participation being associated with academic affiliation, teaching of medical students, having a more specialist clinical practice, and more frequent attendance at multidisciplinary meetings.

It may be that although surgeons are a crucial part of a cancer team, they feel they have a remote role in recruiting patients to medical/oncological trials. The increasing number of neo-adjuvant and peri-operative trials makes this untenable. Furthermore, it can be argued that the surgeon has a pivotal role in influencing patients' expectations about the cancer treatments on offer following surgery. The way the surgeon introduces the idea of trials in general can facilitate any discussions, which follow with the oncologist. If trials are treated as a 'team business', all team members should be made aware of what trials are available for patients in that tumour site and have the skills and knowledge necessary to discuss these trials with patients.

The finding that clinicians at specialist centres are more research oriented than those in DGHs is not surprising. Specialist centres have more staff and resources available for trials and are more likely to be set up to facilitate the running of trials. For example, in a DGH there may be only one histopathologist dealing with several cancer sites and who does not have any protected time to deal with 'trial' tissue blocks in the tight timelines that some trials have. Specialist centres may also be attached to research institutions and therefore their staff is more likely to have a teaching or research focus. Research-oriented clinicians may selfselect into posts within specialist centres or teaching hospitals. It is interesting that clinicians working in specialist centres seem more aware that clinician reluctance could be a barrier to the successful completion of a clinical trial. This may result in clinicians in specialist centres being more aware of their communication training needs and more likely to address their own personal barriers to trial recruitment.

Furthermore, ability to find trial information, and the familiarity of investigators with trials, may differ in different types of hospital. According to one survey, the most common reason cited for physician non-participation in trials was a lack of knowledge about available trials (Taylor, 2004). A review of US publicly funded trials suggested that 'encouraging the development of a user-friendly, transparent, up-to-date, and easily accessible centralised registry could improve both physician and patient awareness of the available trials. In combination with electronic tools, such as, clinical decision support software, a centralised registry could cue physicians to important, applicable clinical trials at the point of care' (Nass et al, 2010, p. 204). A centralised system of trial information available to all clinicians, whatever type of institution they worked in, would potentially overcome some barriers to recruitment in DGHs. 
Lack of clinical trial support has been frequently cited as a barrier to clinical trial involvement (Somkin et al, 2005; Schroen and Brenin, 2008) but this issue does not explain the findings in this study as all the MDTs involved in the study were supported by the Wales Cancer Trials Network whose infrastructure (research nurse time, data management support, etc.) was available to all team members. Other papers refer to lack of reimbursement for clinical trial work and other financial constraints as a barrier to recruitment (Klabunde et al, 2011). Again this was not relevant to this study, which took place in a health service (NHS) where all staff are salaried.

Our hypothesis that clinicians working in breast and colorectal cancer teams would be more research oriented was not borne out by the data. Although these clinicians were more accepting of notreatment arms and more sanguine about patients declining trial entry, they were less likely to seek national or international recognition and had fewer publications compared with clinicians treating other cancer sites. It appears then that specialty by cancer site is not a marker of research orientation. As new therapies are required across all types of cancer, it may be that clinicians specialising in all tumour sites have a broadly equal interest in research, because of the constantly emerging treatments in their field.

The research-orientation scale showed a strong skew towards the non-research orientation end of the scale, with the majority of participants scoring 0 or 1 out of a possible 13. However, despite the skew, in ordinal analyses scores on this scale were significantly different between surgeons and physicians, and between those working in specialist centres and in DGHs, confirming our findings from the single question analyses. This scale can therefore be used in future studies to investigate clinician orientation towards research in general and trials in particular, and could potentially be used to evaluate training materials designed to increase research interest and participation among clinicians.

There are several limitations to this study. The clinicians assessed were all members of recognised cancer MDTs and were involved in regular discussion of patient management. In Wales, all clinical trial recruitment is recorded centrally and MDTs with no recognised trial recruitment were excluded as this was an explicit entry criterion for the associated trial. It is therefore possible that the attitudes expressed are more favourable towards research than would be seen in the general clinical population, as MDTs with no trial experience were excluded.

Second, the questions on attitudes in this study asked primarily about attitudes to RCTs and not to earlier, phase 1 and 2 trials.
Clinicians may have different attitudes to early phase trials, which have different aims and require specialist infrastructure. Third, only a small range of clinician characteristics, such as specialty, were available. Clinician attitudes may also vary by other clinician characteristics, such as number of patients seen per month, clinician sex, training status, previous experience as principal investigator, time since graduation, or academic or industry affiliations (Joffe and Weeks, 2002). In addition, the clinician sample in this study was culturally homogenous and therefore cultural or ethnic differences in attitudes could not be explored. Findings may be different in a more multicultural sample. It is also possible that clinician attitudes may depend on the sociodemographic characteristics of their patient population. Further research in this area would be interesting and may be useful in increasing understanding about current recruitment practices.

In conclusion, this study gives us greater understanding of clinicians' attitudes to clinical trials, in order to target the problem of low and selective accrual. Despite an increase in overall recruitment to trials over the past 5 years, patients' understanding of them and health professionals' explanations about them are still problematic. There is a crucial need for all cancer team members to be fully supportive and aware of the trials that are available in their centres, and for all to receive and deliver consistent information to patients and their relatives. The results of this study are part of a larger project, in which members from participating MDTs have taken part in training. The training has addressed problems such as team dynamics acting as a barrier to trial recruitment, inconsistent team communication, and the difficulty of talking about trials where patients are unenthusiastic about joining for different reasons. Previous research has shown that communication skills training can also alter the attitudes and beliefs of clinicians (Jenkins and Fallowfield, 2002). The overall aims of this work are to increase the number of patients offered a trial by clinicians, and to increase the quality of communication about trials when they are discussed.

\section{ACKNOWLEDGEMENTS}

We thank all the clinicians who gave up their time to take part, Cancer Research UK for funding, and the Wales Cancer Trials Network (now a part of the National Institute for Social Care and Health Research Clinical Research Centre) for their help with this study.

\section{REFERENCES}

Antman K, Amato D, Wood W, Corson J, Suit H, Proppe K, Carey R, Greenberger J, Wilson R, Frei E (1985) Selection bias in clinical-trials. J Clin Oncol 3: 1142-1147

Caldwell PHY, Craig JC, Butow PN (2005) Barriers to Australian physicians' and paediatricians' involvement in randomised controlled trials. Med J Aust 182: 59-62

Castel P, Negrier S, Boissel J-P (2006) Why don't cancer patients enter clinical trials? A review. Eur J Cancer 42: 1744-1748

Christian MC, Trimble EL (2003) Increasing participation of physicians and patients from underrepresented racial and ethnic groups in national cancer institute-sponsored clinical trials. Cancer Epidemiol Biomarkers Prev 12: $277 \mathrm{~s}-283 \mathrm{~s}$

Cottin V, Arpin D, Lasset C, Cordier JF, Brune J, Chauvin F, Trillet-Lenoir V (1999) Small-cell lung cancer: Patients included in clinical trials are not representative of the patient population as a whole. Ann Oncol 10: $809-815$

D’Agostino Snr RB (2005) Principal components analysis. In: Armitage P, Colton T (eds) Encyclopedia of Biostatistics. 2nd edn, pp 4224-4232. Wiley: Chichester

EDICT (2008) The EDICT Project: Policy Recommendations to Eliminate Disparities in Clinical Trials. EDICT Project: Houston, TX
Ellis PM, Butow PN, Simes RJ, Tattersall MHN, Dunn SM (1999) Barriers to participation in randomized clinical trials for early breast cancer among Australian cancer specialists. Aust NZ J Surg 69: 486-491

Elting LS, Cooksley C, Bekele BN, Frumovitz M, Avritscher EBC, Sun C, Bodurka DC (2006) Generalizability of cancer clinical trial results. Cancer 106: $2452-2458$

Fallowfield L, Ratcliffe D, Souhami R (1997) Clinician's attitudes to clinical trials of cancer therapy. Eur J Cancer 33: 2221-2229

Fayter D, McDaid C, Eastwood A (2007) A systematic review highlights threats to validity in studies of barriers to cancer trial participation. J Clin Epidemiol 60: 990 - 1001

Grunfeld E, Zitzelberger L, Coristine M, Aspelund F (2002) Barriers and facilitators to enrollment in cancer clinical trials: qualitative study of the perspectives of clinical research associates. Cancer 95: 1577-1583

Hjorth M, Holmberg E, Rodjer E, Taube A, Westin J (1996) Physicians' attitudes toward clinical trials and their relationship to patient accrual in a nordic multicenter study on myeloma. Control Clin Trials 17: $372-386$

Hjorth M, Holmberg E, Rodjer S, Westin J, Fagerberg B, Magnusson B, Willstrand M, Magnus C, Lennartsson J, Svensson R, Hellqvist L, Langer L, Hoffman P, Larsson S, Olsson S, Rindner A, Evaldsson U, Olsson K, Grande B, Lindqvist O, Blom HL, Sarg M (1992) Impact of active and 
passive exclusions on the results of a clinical-trial in multiple-myeloma. Br J Haematol 80: 55-61

Jenkins V, Fallowfield L (2002) Can communication skills training alter physicians' beliefs and behavior in clinics? J Clin Oncol 20: 765-769

Jenkins V, Farewell D, Batt L, Maughan T, Branston L, Langridge C, Parlour L, Farewell V, Fallowfield L (2010) The attitudes of 1066 patients with cancer towards participation in randomised clinical trials. Br J Cancer 103: $1801-1807$

Jenkins V, Leach L, Fallowfield L, Nicholls K, Newsham A (2002) Describing randomisation: patients' and the public's preferences compared with clinicians' practice. $\mathrm{Br} J$ Cancer 87: 854-858

Joffe S, Weeks JC (2002) Views of American oncologists about the purposes of clinical trials. J Natl Cancer Inst 94: 1847-1853

Kaas R, Hart AAM, Rutgers EJT (2005) The impact of the physician on the accrual to randomized clinical trials in patients with primary operable breast cancer. Breast 14: 310-316

Klabunde CN, Keating NL, Potosky AL, Ambs A, He Y, Hornbrook MC, Ganz PA (2011) A population-based assessment of specialty physician involvement in cancer clinical trials. J Natl Cancer Inst 103: 384-397

Lally F, Crome P (2010) The PREDICT study: increasing the participation of the elderly in clinical trials: the opinions of medical and associated professionals. Keele University: Accessed. 3rd March 2011 at http:// www.predicteu.org/Reports/PREDICT_WP2_Final_Report_V5.pdf

Maslin-Prothero S (2006) The role of the multidisciplinary team in recruiting to cancer clinical trials. Eur J Cancer Care 15: 146-154

McCulloch P, Kaul A, Wagstaff GF, Wheatcroft J (2005) Tolerance of uncertainty, extroversion, neuroticism and attitudes to randomized controlled trials among surgeons and physicians. Br J Surg 92: 1293-1297

Murthy VH, Krumholz HM, Gross CP (2004) Participation in cancer clinical trials: Race-, Sex-, and age-based disparities. JAMA 291: 2720-2726

Nass SJ, Moses HL, Mendelsohn J (eds) (2010) A National Cancer Clinical Trials System for the 21st Century: Reinvigorating the NCI Cooperative Group Program. Committee On Cancer Clinical Trials and the NCI Cooperative Group Program; Institute of Medicine

Rahman ZU, Frye DK, Buzdar AU, Smith TL, Asmar L, Champlin RE, Hortobagyi GN (1997) Impact of selection process on response rate and long-term survival of potential high-dose chemotherapy candidates treated with standard-dose doxorubicin-containing chemotherapy in patients with metastatic breast cancer. J Clin Oncol 15: 3171-3177
Robinson EJ, Kerr CEP, Stevens AJ, Lilford RJ, Braunholtz DA, Edwards SJ, Beck SR, Rowley MG (2005) Lay public's understanding of equipoise and randomisation in randomised controlled trials. Health Technol Assess 9: 8

Schroen AT, Brenin DR (2008) Clinical trial priorities among surgeons caring for breast cancer patients. Am J Surg 195: 474-480

Seifer SD, Michaels M, Collins S (2010) Applying community-based participatory research principles and approaches in clinical trials: forging a new model for cancer clinical research. Prog Comm Health Partnerships Res Edu Action 4: 37-46

Siminoff LA, Zhang A, Colabianchi N, Sturm CMS, Shen QS (2000) Factors that predict the referral of breast cancer patients onto clinical trials by their surgeons and medical oncologists. J Clin Oncol 18: 1203-1211

Somkin CP, Altschuler A, Ackerson L, Geiger AM, Green SM, Mouchawar J, Holup J, Fehrenbacher L, Nelson A, Glass A, Polikoff J, Tishler S, Schmidt C, Field T, Wagner E (2005) Organizational barriers to physician participation in cancer clinical trials. Am J Manag Care 11: 403-421

Taylor H (2004) Most physicians do not participate in clinical trials because of lack of opportunity, time, personnel support and resources. Harris Interactive: Health Care News 4: 1-8

Taylor KM, Kelner M (1987) Interpreting physician participation in randomised clinical trials: the physician orientation profile. $J$ Health Social Behav 28: 389-400

Tejeda HA, Green SB, Trimble EL, Ford L, High JL, Ungerleider RS, Friedman MA, Brawley OW (1996) Representation of African-americans, hispanics, and whites in national cancer institute cancer treatment trials. J Natl Cancer Inst 88: 812-816

Tournoux C, Katsahian S, Chevret S, Levy V (2006) Factors influencing inclusion of patients with malignancies in clinical trials: a review. Cancer 106: $258-278$

UK Clinical Research Network (2010) UK Clinical Research Network: Portfolio Database. http://public.ukcrn.org.uk/Search/Portfolio.aspx? level1 $=1$

Weinberg AD, Cooper HP, Mejia NI, Spiker CA (2004) Attitudes of primary care physicians and specialists about cancer clinical trials: a survey of Texas physicians. Texas Med 100: 66-72

White C, Gilshenan K, Hardy J (2008) A survey of the views of palliative care healthcare professionals towards referring cancer patients to participate in randomized controlled trials in palliative care. Support Care Cancer 16: $1397-1405$ 